\title{
CASO -2016: Adulto eutiroideo con oftalmopatía de Graves unilateral: reporte de caso y revisión de tema
}

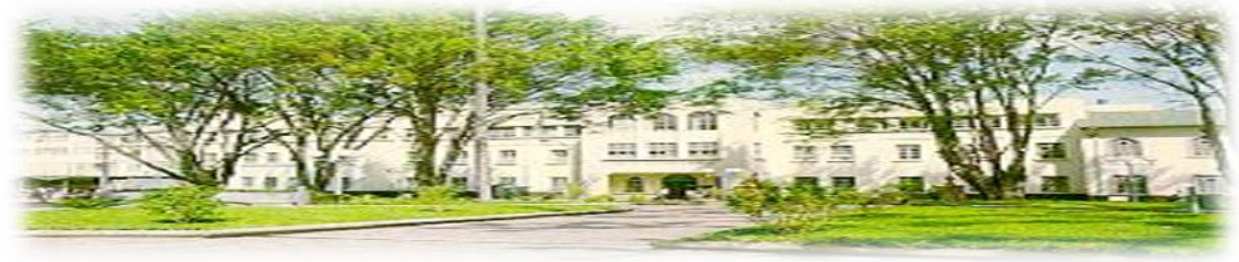

Hospital San Juan de Dios, San José, Costa Rica. Fundado en 1845

Recibido:

$09 / 12 / 2016$

Aceptado:

$12 / 12 / 2016$

Pablo Coste Murillo ${ }^{1}$

Olger López Salas ${ }^{2}$

${ }^{1}$ Médico Especialista en Medicina Interna, Residente de Gastroenterología. Programa de Estudios de Postgrado UCR- CENDEISSS. Hospital San Juan de Dios. Correo electrónico: costepablo@gmail.com

${ }^{2}$ Médico Especialista en Endocrinología. Programa de Estudios de Posgrado UCR-CENDEISSS. Hospital San Juan de Dios. Correo electrónico: olopezsalas@ gmail.com

\section{RESUMEN}

La oftalmopatía tiroidea o de Graves es una condición que afecta la órbita, en la cual la reacción inmune a un autoantígeno, probablemente el receptor de TSH, provoca una reacción inflamatoria en los músculos extraoculares y el tejido orbitario $^{1}$. Sólo un pequeño porcentaje de los pacientes con oftalmopatía presenta una condición eutiroidea y un porcentaje aún menor, afección unilateral o asimétrica ${ }^{1}$. La historia natural de la oftalmopatía unilateral es desconocida ${ }^{2}$. Presentamos el reporte de caso y revisión de tema de un paciente eutiroideo con oftalmopatía tiroidea unilateral.

\section{PALABRAS CLAVE}

Oftalmopatía tiroidea; oftalmopatía de Graves
Graves' or thyroid ophthalmopathy is a condition confined to the orbit in which an immune reaction to an autoantigen, which is probably the TSH receptor, causes an inflammatory reaction affecting the extraocular muscles and orbital tissue ${ }^{1}$. Just a small percentage of patients with ophtalmophaty are euthyroid and even a smaller percentage, have unilateral or asymmetrical involvement. The natural history of unilateral TAO is unknown ${ }^{2}$. This is a case report and review of an euthyroid patient with unilateral thyroid ophthalmopathy

\section{KEY WORDS}

Thyroid ophthalmopathy, Graves ophthalmopathy

\section{ABSTRACT}




\section{CASO CLÍNICO}

Masculino de 65 años hipertenso, diabético tipo 2 y dislipidémico, con antecedente de hipotiroidismo en hermana y bocio en madre, quien consulta por cuadro de un año de epífora en ojo derecho y proptosis ipsilateral progresiva. Aúna visión borrosa y diplopía en los últimos dos meses previos a consultar a un médico.

Acude a un servicio de oftalmología en donde se realiza un TAC de cráneo y órbitas el cual evidenció globos oculares de tamaño, forma y estructura normal, con grasa retrocular normal, músculo recto inferior medial y superior derechos con vientres prominentes de hasta $9 \mathrm{~mm}$ de grosor, con inserciones tendinosas de grosor normal y músculos extrínsecos izquierdos con grosor y atenuación normal. Se complementa los estudios con unas pruebas de función tiroidea que evidenciaron la TSH y T4 dentro de rangos normales.

Ante la sospecha de una endocrinopatía, es referido a endocrinología. Es valorado y presenta exoftalmos del ojo derecho de $25 \mathrm{~mm}$, quemosis y blefaritis ipsilateral y una tiroides palpable sin nódulos. Se repiten las pruebas tiroideas las cuales se encentran dentro de rango normal. Se solicitan los anticuerpos antitiroideos los cuales son negativos y los antirecptor de tiroptropina los cuales se encuentran levemente elevados.

Se concluye que se trata de una oftalmopatía tiroidea unilateral, en un paciente eutiroideo y con una Escala Clínica de Actividad de 4pts. Se inicia metilprednisolona 500mg IV cada semana \#6 y luego $250 \mathrm{mg}$ cada semana \#6. La evolución es satisfactoria y el paciente cursa asintomático y sin esteroides por lo cual se realiza cirugía estética. $\mathrm{Al}$ año de la cirugía el paciente cursa asintomático y sin necesidad de tratamiento.

\section{DISCUSIÓN Y ANÁLISIS DEL CASO}

\section{Generalidades}

La oftalmopatía de Graves, también llamada oftalmopatía tiroidea, es una enfermedad ocular con potencial riesgo de ceguera que ha planteado un reto médico durante más de dos siglos ${ }^{3}$. Dicha patología no ocurre sólo en el contexto de la enfermedad de Graves- Basedow, ha sido descrita asociada a tiroiditis subaguda, cáncer de tiroides y hasta en un $10 \%$ con otras formas de enfermedad tiroidea crónica autoinmune ${ }^{4}$.

Si bien esta entidad se asocia usualmente al hipertiroidismo por Graves, la relación temporal con la afección tiroidea no es consistente y se ha estimado que el $77 \%$ de los pacientes son hipertiroideos, un $21 \%$ eutiroideos y un $2 \%$ hipotiroideos ${ }^{5}$.

Cuando se asocia a hipertiroidismo, el intervalo promedio entre el diagnóstico clínico y la oftalmopatía es de 3.3 años en hombres y 3.6 en mujeres. En estos pacientes, dos terceras partes presentan síntomas oculares en los 18 meses posteriores al diagnóstico del hipertiroidismo, mientras que el $20 \%$ presenta síntomas antes y el $40 \%$ concomitantemente con el diagnóstico ${ }^{5,6}$. Sin embargo, la proptosis unilateral como resultado de la enfermedad de Graves puede ocurrir hasta 20 a 30 años después de la afección tiroidea y en el caso de hipotiroidismo, un estudio sugiere intervalos de hasta 15 años?

La afección ocular es usualmente bilateral, sin embargo, puede ser asimétrica o unilateral hasta en un $10-15 \%^{2}$. Diversos reportes sugieren que la enfermedad unilateral puede ser bilateral desde el inicio sin ser clínicamente aparente o lateralizarse en al menos la mitad de los casos ${ }^{2}$. La afección unilateral persistente es rara y ha sido descrita solamente en casos control ${ }^{2}$.

\section{Epidemiología}

La oftalmopatía tiroidea presenta una incidencia ajustada anual de 16 mujeres y 3 hombres por 100 000 habitantes, con el pico de incidencia en la cuarta y quinta décadas, aunque también se puede presentar en niños y ancianos ${ }^{3,8}$.

\section{Patogenia}

Se postula que la oftalmopatía de Graves es iniciada por linfocitos $\mathrm{T}$ autorreactivos en contra de antígenos compartidos por la tiroides y la órbita ${ }^{9}$.

Estos inician una cascada inflamatoria que incluye la secreción de citokinas, la estimulación de la proliferación de fibroblastos orbitales, la expansión del tejido adiposo y la secreción de glicosaminoglucanos hidrofílicos por los fibroblastos ${ }^{9}$.

Los linfocitos B también están involucrados como células presentadoras de antígenos y productoras 
de anticuerpos ${ }^{9}$. Se han planteado al receptor de tirotropina y el receptor del factor de crecimiento similar a la insulina como los autoantígenos compartidos 9 . Entre otros factores involucrados, se han sugerido determinantes genéticos los cuales son poco comprendidos y algunos factores ambientales que aparentan tener un papel en el desarrollo y progresión de la oftalmopatía sin ser concluyentes ${ }^{9}$.

\section{Sintomatología}

Diversas series de casos de centros de referencia indican que la oftalmopatía tiroidea clínicamente manifiesta ocurre en alrededor del $50 \%$ de los casos de enfermedad de Graves, es relevante en un $20-30 \%$ y presenta riesgo de ceguera debido a neuropatía óptica, afección corneal o ambas en un $3-5 \%$.

Generalmente hay una fase inflamatoria inicial (activa) que dura 2 años, seguida por una fase de estabilización (meseta) y eventualmente una de remisión (inactiva), sin embargo, los síntomas oculares pueden progresar, mantenerse o mejorar espontáneamente ${ }^{9}$.

Los síntomas comunes incluyen fotofobia, lagrimeo, dolor, diplopia, sensación de arena en el ojo, y sensación de opresión ocular. Los signos más comunes incluyen proptosis, retracción del párpado superior, edema y eritema periorbitario y conjuntival. Como se mencionó anteriormente, aproximadamente un 5\% presenta dolor intenso, inflamación, ulceración corneal y neuropatía óptica con importante riesgo de ceguera ${ }^{3,9}$.

La afección subclínica es común; en aproximadamente un $70 \%$ de pacientes con oftalmopatía tiroidea se evidencia engrosamiento de los músculos extraoculares por TAC o RMN. En los casos de afección unilateral, la imagen orbital generalmente corrobora la afección asimétrica ${ }^{3}$.

\section{Diagnóstico}

El diagnóstico es clínico. Deben descartarse anormalidades en las pruebas de función tiroidea, sin embargo y como se explicó previamente, dicha patología puede presentarse en el hipertiroidismo, hipotiroidismo o eutiroidismo ${ }^{10}$.
La medición de los anticuerpos antireceptor de tirotropina, puede tener importante valor diagnóstico dada su alta sensibilidad y especificidad para enfermedad de Graves ${ }^{9}$. Estos anticuerpos parecen ser útiles en predecir el curso de la enfermedad y la respuesta a tratamiento, sin embargo se desconoce si son predictores del desarrollo de la oftalmopatía tiroidea ${ }^{11}$.

Cuando el diagnóstico es incierto, las imágenes orbitales con TAC y/o RMN son necesarias. Estas suelen mostrar engrosamiento de los músculos extraoculares sin afección de los tendones y/o aumento del tejido fibroadiposo orbital ${ }^{9}$.La TAC evidencia afección contralateral hasta en un 50-90\% de los pacientes con aparente afección clínica unilateral $^{12}$.

\section{Evaluación}

La Escala Clínica de Actividad modificada, CAS por sus siglas en inglés, constituye la escala de evaluación más frecuentemente utilizada en la orbitopatía tiroidea. Esta asigna un punto a cada síntoma y signo presente (dolor retrobulbar, dolor evocado ante la fijación de la mirada, eritema palpebral, inyección conjuntival, quemosis, inflamación de la carúncula y edema palpebral ${ }^{8}$.

Aunque imperfecta, esta escala ha demostrado ser predictiva para la respuesta a la terapia inmunosupresora, sin embargo no para la progresión y severidad de la oftalmopatía tiroidea ${ }^{9}$.

\section{Diagnóstico diferencial}

En los raros casos de oftalmopatía unilateral, el diagnóstico diferencial debe incluir masas introrbitales, fístulas carotídeas cavernosas, pseudotumor orbitario, celulitis orbitaria, trombosis de senos cavernosos y causas de pseudoexoftalmos como miopía anisometrópica magna ${ }^{5,13,14}$.

\section{Manejo}

Las medidas iniciales incluyen evitar la exposición a fumado, estrés, alimentos ricos en sal y glutamato monosódico, luces fluorescentes y calor $\operatorname{seco}^{8}$.

Toda disfunción tiroidea debe corregirse ${ }^{9,15}$. En estudios randomizados se ha evidenciado que la 
terapia con radioyodo para pacientes con enfermedad de Graves puede provocar la progresión de la oftalmopatía hasta en un $15 \%$ de los pacientes, por ende, debe tenerse en cuenta para el seguimiento de estos pacientes ${ }^{16}$.

La orbitopatía tiroidea leve usualmente sólo requiere medidas locales (lubricantes, ungüentos, lentes oscuros y prismas para reducir la diplopía $)^{9}$.

Los pacientes con riesgo de ceguera por neuropatía óptica distiroidea, ameritan tratamiento esteroideo inmediato. A pesar de que no hay un esquema de tratamiento establecido, el régimen de $1 \mathrm{~g}$ de metilprednisolona por tres días es comúnmente utilizado. El tratamiento subsecuente depende de la respuesta. Si no hay mejoría posterior a una o dos semanas, los pacientes deben someterse a una descompresión orbitaria quirúrgica ${ }^{17}$.

Para los casos moderados a severos, las altas dosis de esteroides orales (40mg o más) son comúnmente utilizadas, así como metilprednisolona para los casos más severos $(0.5 \mathrm{~g}$, luego $0.25 \mathrm{~g}$ c/semana por seis semanas cada uno). Una vez alcanzada la mejoría, se inicia el desescalonamiento hasta suspender a los cuatro a seis meses. En varias series de casos de pacientes tratados con esteroides orales, se ha observado una respuesta general de alrededor de un $63 \%$. Cuando la elección es la terapia intravenosa debe utilizarse bajo supervisión estricta $^{17}$.

La irradiación orbitaria puede brindar un beneficio a la terapia, particularmente en los casos en los que hay afección de la motilidad ocular ${ }^{18}$.

Cuando la oftalmopatía haya cumplido seis meses de inactividad clínica, la cirugía para corregir la retracción palpebral puede ayudar a aminorar las alteraciones estéticas ${ }^{17}$.

\section{CONCLUSIONES}

La oftalmopatía de Graves unilateral constituye una patología poco frecuente, pero con severas consecuencias en caso de un diagnóstico tardío. Su presentación en el contexto de un eutiroidismo es aún más infrecuente por lo cual exige una alta sospecha diagnóstica por parte del médico tratante. En estos casos, la sintomatología ocular y los hallazgos radiológicos pueden ser la única pista para un diagnóstico y tratamiento oportuno. Se expuso el primer caso reportado en la literatura costarricense con el fin de promover el conocimiento e incentivar la investigación en el tema.

\section{BIBLIOGRAFÍA}

1. Gómez JM. Valoración y tratamiento de la oftalmopatía de Graves. Endocrinol Nutr. 2004;51:60.

2. Ch. Daumerie, Th. Duprez, A. Boschi. Long-term multidisciplinary follow-up of unilateral thyroid-associated orbitopathy European Journal of Internal Medicine 19 (2008) 531-536.

3. Bahn R. Graves' Ophthalmopathy. NEJM 362;8, 2010.

4. Kymberly P, Cockerham, Stephanie SC. Thyroid eye disease. Neurol Clin. 2010;28:729---755.

5. N Kamminga, N M Jansonius, J W R Pott, et al. Unilateral proptosis: the role of medical history. $\mathrm{Br} \mathrm{J}$ Ophthalmol. 2003 Mar; 87(3): 370-371.

6. Bartley GB, Fatourechi V, Kadrmas EF, et al. Chronology of Graves' ophthalmopathy in an incidence cohort. Am J Ophthalmol 1996; 121:426.

7. Brownlie BEW, Newton OAG, Singh SP. Ophthalmopathy associated with primary hypothyroidism. Acta Endocrinol 1975;79:691-699.

8. K Cockerham, S Chan. Thyroid Eye Disease. Neurol Clin 28 (2010) 729-755.

9. Bartalena L, Tanda ML. Graves' $O p$ hthalmopathy. NEJM 360;10, 2009.

10. Kendler DL, Lippa J, Rootman J. The initial clinical characteristics of Graves' orbitopathy vary with age and sex. Arch Ophthalmol 1993;111:197-201.

11. Stan M, Bahn Rebecca. Risk Factors for Development or Deterioration of Graves' Ophtalmopathy. Thyroid, Vol 2o, Number 7, 2010.

12. Burch HB, Wartofsky L. Graves' ophthalmopathy: current concepts regarding pathogenesis and management. Endocr Rev 1993;14:747-793.

13. Fernández RV. Manifestaciones clínicas de la oftalmopatía tiroidea. An Sist Sanit Navar. 2008;31 Suppl. 3:45---56. 
14. Perez JV, Coloma JE, Prada MC. Orbitopatía tiroidea (fisiopatología, diagnóstico y tratamiento). Arch Soc Esp Oftalmol. 2003;78:407---431.

15. Pfeilschifter J, Ziegler R. Smoking and endocrine ophthalmopathy: impact of smoking severity and current vs. lifetime cigarette consumption. Clin Endocrinol (Oxf) 1996;45:477-481.

16. Bartalena L, Marcocci C, Bogazzi F, et. al. Relation between therapy for hyperthyroidism and the course of Graves' ophthalmopathy. N Engl J Med 1998;338: 73-78.

17. Consensus statement of the European Group on Graves' Orbitopathy (EUGOGO) on management of Graves' orbitopathy. Thyroid 2008;18:333-346.

18. Mourits MP, van Kempen-Harteveld ML, Garcia MB, et al. Radiotherapy for Graves' orbitopathy: randomized placebo-controlled study. Lancet 2000;355:1505-1509.

\section{CONFLICTO DE INTERÉS Y/O AGRADE- CIMIENTOS}

Los autores declaran que no existió ningún conflicto de interés en el presente reporte. 
ANEXOS
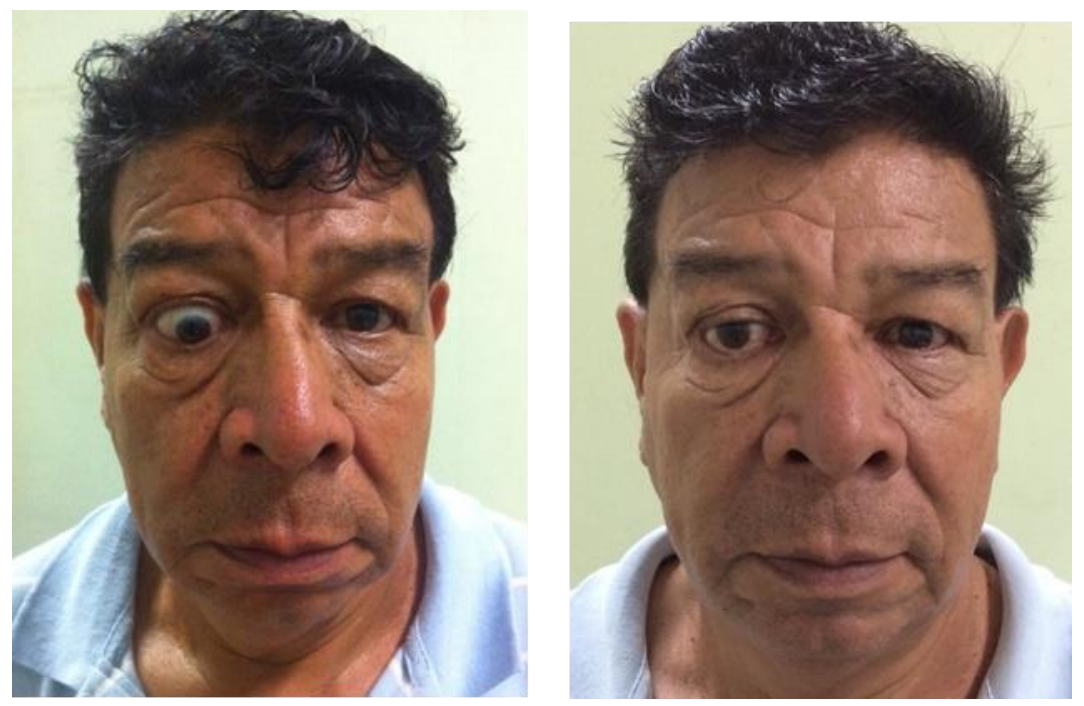

Figura 1: vista frontal de la oftalmopatía tiroidea pre tratamiento (izq) y postratamiento (der).

Fuente: HSJD
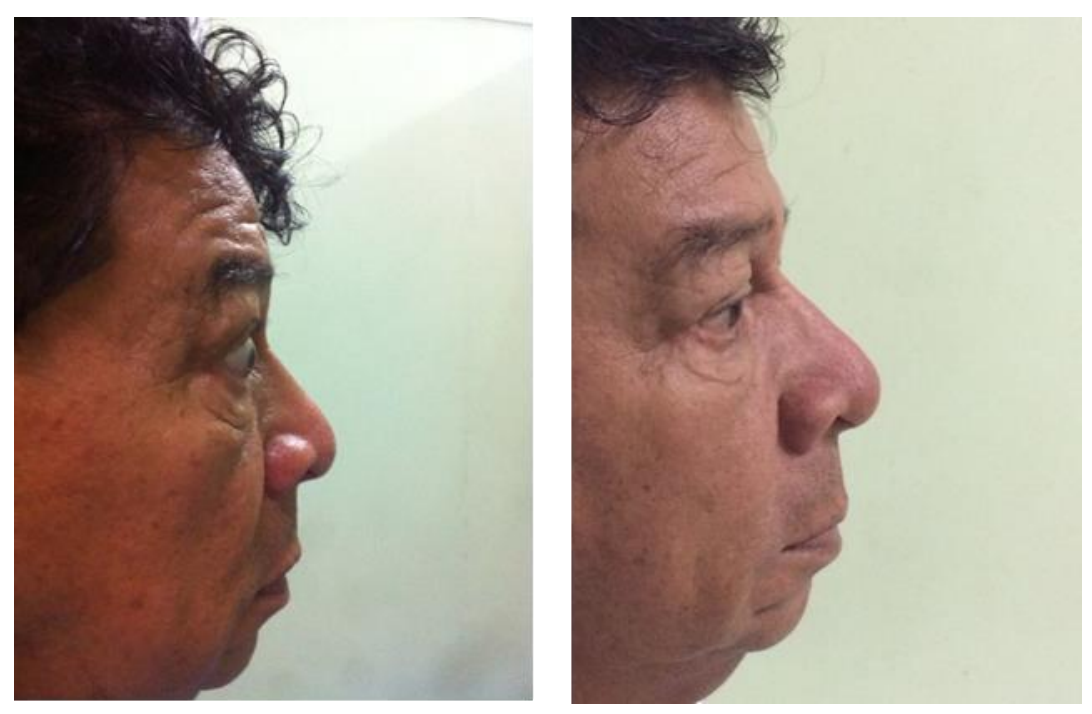

Figura 2: vista lateral de la oftalmopatía tiroidea pre tratamiento (izq) y postratamiento (der).

Fuente: HSJD 


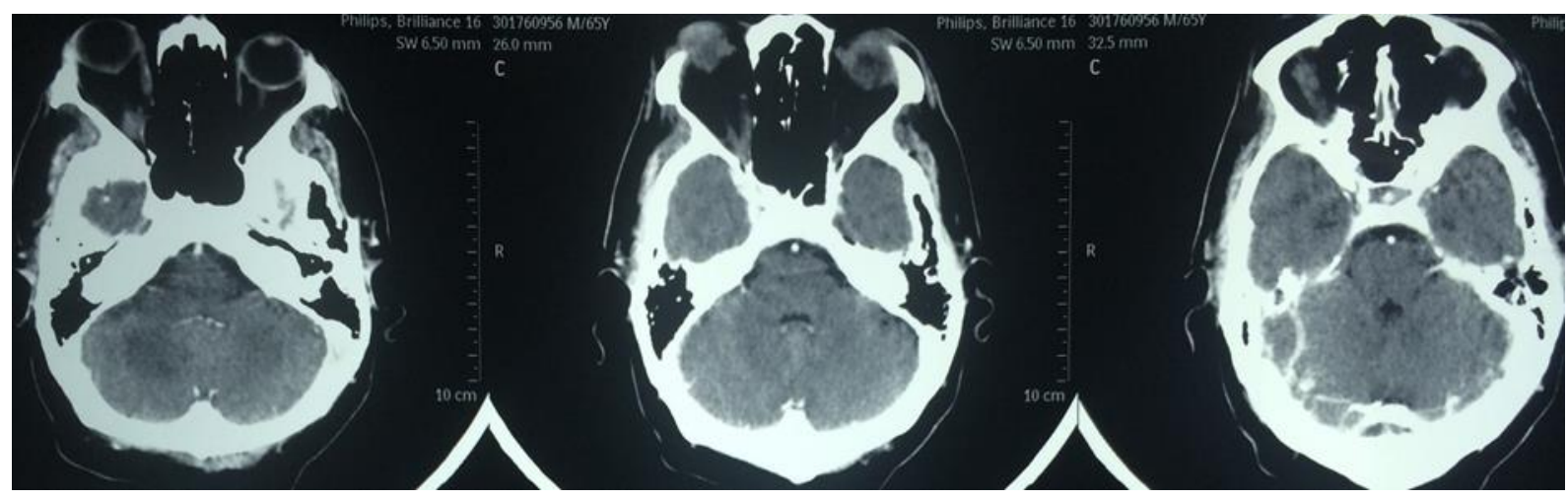

Figura 3: corte de TAC de SNC que evidencia engrosamiento unilateral de los músculos extraoculares derechos, sin afección de los tendones

Fuente: HSJD 